\title{
JTLV: A Framework for Developing Verification Algorithms ${ }^{\star}$
}

\author{
Amir Pnueli, Yaniv Sa' ${ }^{1}{ }^{1}$, and Lenore D. Zuck ${ }^{2}$ \\ 1 Weizmann Institute of Science \\ yaniv.saar@weizmann.ac.il \\ ${ }^{2}$ University of Illinois at Chicago \\ lenoreacs.uic.edu
}

\section{Introduction}

JTLV 11 is a computer-aided verification scripting environment offering state-of-the-art Integrated Developer Environment for algorithmic verification applications. JTLV may be viewed as a new, and much enhanced TLV [18], with Java rather than TLV-basic as the scripting language. JTLV attaches its internal parsers as an Eclipse editor, and facilitates a rich, common, and abstract verification developer environment that is implemented as an Eclipse plugin.

JTLV allows for easy access to various low-level BDD packages with a high-level Java programming environment, without the need to alter, or even access, the implementation of the underlying BDD packages. It allows for the manipulation and on-the-fly creation of BDD structures originating from various BDD packages, whether existing ones or userdefined ones. In fact, the developer can instantiate several BDD managers, and alternate between them during run-time of a single application so to gain their combined benefits.

Through the high-level API the developer can load into the Java code several SMVlike modules representing programs and specification files, and directly access their components. The developer can also procedurally construct such modules and specifications, which enables loading various data structures (e.g., statecharts, LsCs, and automata) and compile them into modules.

JTLV offers users the advantages of the numerous tools developed by Java's ecosystem (e.g., debuggers, performance tools, etc.). Moreover, JTLV developers are able to introduce software methodologies such as multi-threading, object oriented design for verification algorithms, and reuse of implementations.

\section{JTLV: Architecture}

JTLV, described in Fig. 1] is composed of three main components, the API, the Eclipse Interface, and the Core.

\footnotetext{
* This research was supported by the John von Neumann Minerva Center for the Development of Reactive Systems at the Weizmann Institute of Science, and by an Advanced Research Grant awarded to David Harel from the European Research Council (ERC) under the European Community's 7th Framework Programme (FP7/2007-2013). This material is based on work supported by the National Science Foundation, while Lenore Zuck was working at the Foundation. Any opinion, finding, and conclusions or recommendations expressed in this article are those of the author and do not necessarily reflect the views of the National Science Foundation.

${ }^{1}$ JTLV homepage: http: / / jtlv.ysaar.net
}

T. Touili, B. Cook, and P. Jackson (Eds.): CAV 2010, LNCS 6174, pp. 171 174, 2010.

(C) Springer-Verlag Berlin Heidelberg 2010 


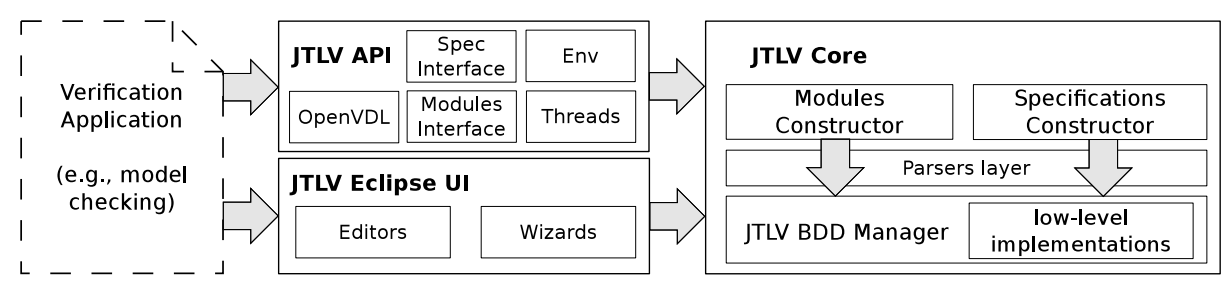

Fig. 1. JTLV Architecture

API. In the following we present a set of sample functionalities. An exhaustive list of the API is in [20]

- TLV-like ability to procedurally create and manipulate BDD's on-the-fly, a useful feature when dealing with abstractions and refinements ([1]);

- seamlessly alternate BDD packages at run-time (due to the factory design pattern [22]);

- save (and load) BDDs to (and from) the file system;

- load modules written in NuSMV-like language enriched with Cadence SMV-like parsing of loops and arrays of processes;

- procedurally access module's fields as well as its BDD's;

- perform basic functionalities on a module, e.g., compute successors or predecessors, feasible states, shortest paths from one state to another, etc.;

- procedurally create new modules and add, remove, and manipulate fields ;

- load temporal logic specification files;

- procedurally create and access the specification objects.

JTLV supports threads, that are Java native threads coupled with dedicated BDD memory managers. Each thread can execute freely, without dependencies or synchronization with other threads. To allow for BDD-communication among threads, JTLV provides a low-level procedure that copies BDDs from one BDD manager into another. Our experience has shown that for applications that accommodate compositionality, an execution using threads outperforms its sequential counterparts.

Assisted by the API, the user can implement numerous types of verification algorithms, some mentioned in the next section. It also contains the OpenVDL (Open Verification Developer Library), which is a collection of known implementations enabling their reuse.

Eclipse User Interface. Porting the necessary infrastructure into Java enables plugging JTLV into Eclipse, which in turn facilitates rich new editors to module and specification languages (see website for snapshots). A new JTLV project automatically plugs-in all libraries. JTLV project introduces new builders that take advantage of the underlying parsers, and connects them to these designated new editors.

Core. The core component encapsulates the BDD implementation and parses the modules and specifications. Through the JAVA-BDD ([22]) factory design pattern, a developer can use a variety of BDD packages (e.g., CUDD [21], BUDDY [14], and CAL [19]), or design a new one. This also allows for the development of an application regardless of the BDD package used. In fact, the developer can alternate BDD packages during 
run-time of a single application. The encapsulation of the memory management system allows JTLV to easily instantiate numerous BDD managers so to gain the combined benefits of several BDD packages simultaneously. This is enabled by APIs that allow for translations among BDD's generated by different packages, so that one can apply the functionality of a BDD-package on a BDD generated by another package.

\section{Conclusion, Related, and Future Work}

We introduced JTLV, a scripting environment for developing algorithmic verification applications. JTLV is not a dedicated model checker (e.g. [2 13[10]) - its goal is to provide for a convenient development environment, and thus cannot be compared to a particular model checkers. Yet, as shown in Table 1] our implementation of invariance checking at times outperforms similar computations in such model checkers.

Table 1. Performance results (in sec.) of JTLV, compared to other model checkers

\begin{tabular}{|c||c||c||c|}
\hline Check Invariant & Muxsem 56 & Bakery 7 & Szymanski 6 \\
\hline \hline JTLV & 11 & 39.9 & 34.4 \\
\hline TLV & 21.4 & 36.2 & 19 \\
\hline NuSMV & 18.1 & 37.8 & 19.4 \\
\hline Cadence SMV & 24.6 & 53.6 & 36.7 \\
\hline
\end{tabular}

We are happy to report that JTLV already has a small, and avid, user community, including researchers from Imperial College London [15], New York University [5 46], Bell Labs Alcatel-Lucent [5|46], Weizmann Institute [89], Microsoft Research Cambridge, RWTH-Aachen, California Institute of Technology [24]23], GRASP Laboratory University of Pennsylvania [7], and University of California Los Angeles. In these works JTLV is applied to: Streett and Rabin Games; Synthesis of GR(k) specifications; Compositional multi-theaded model checking; Compositional LTL model checking; Verifying heap properties; Automata representation of LSCs and Statecharts; Synthesis of LSCs and of hybrid controllers.

The JTLV library (see [20]) includes numerous model checking applications, including LTL and CTL* model checking [12], fair-simulation [11], a synthesis algorithm [17], Streett and Rabin games [16], compositional model checking ([3]), and compositional multi threaded model checking [6]. The API can also facilitate the reduction of other models into the verification framework (see, e.g., [8] where LSCs are reduced to automata).

We are currently developing a new thread-safe BDD package to allow concurrent access from multiple clients. Integrating a thread-safe BDD package into JTLV will entail a new methodology, which will streamline the development of multi-threaded symbolic algorithms. This calls for an in-depth overview of many symbolic applications. We are also in the process of developing new interfaces to non-BDD managers

\section{References}

1. Balaban, I., Fang, Y., Pnueli, A., Zuck, L.D.: IIV: An Invisible Invariant Verifier. In: Etessami, K., Rajamani, S.K. (eds.) CAV 2005. LNCS, vol. 3576, pp. 408-412. Springer, Heidelberg (2005) 
2. Cimatti, A., Clarke, E.M., Giunchiglia, F., Roveri, M.: NuSMV: A new symbolic model verifier. In: Halbwachs, N., Peled, D.A. (eds.) CAV 1999. LNCS, vol. 1633, pp. 495-499. Springer, Heidelberg (1999)

3. Cohen, A., Namjoshi, K.S.: Local proofs for linear-time properties of concurrent programs. In: Gupta, A., Malik, S. (eds.) CAV 2008. LNCS, vol. 5123, pp. 149-161. Springer, Heidelberg (2008)

4. Cohen, A., Namjoshi, K.S., Sa'ar, Y.: A dash of fairness for compositional reasoning. In: Touili, T., Cook, B., Jackson, P. (eds.) CAV 2010. LNCS, vol. 6174, pp. 543-557. Springer, Heidelberg (2010)

5. Cohen, A., Namjoshi, K.S., Sa'ar, Y.: Split: A compositional LTL verifier. In: Touili, T., Cook, B., Jackson, P. (eds.) CAV 2010. LNCS, vol. 6174, pp. 558-561. Springer, Heidelberg (2010)

6. Cohen, A., Namjoshi, K.S., Sa'ar, Y., Zuck, L.D., Kisyova, K.I.: Parallelizing a symbolic compositional model-checking algorithm (in preparation) (2010)

7. Gazit, H.K., Ayanian, N., Pappas, G., Kumar, V.: Recycling controllers. In: IEEE Conference on Automation Science and Engineering, Washington (August 2008)

8. Harel, D., Maoz, S., Segall, I.: Using automata representations of LSCs for smart play-out and synthesis (in preparation) (2010)

9. Harel, D., Segall, I.: Synthesis from live sequence chart specifications (in preparation) (2010)

10. Holzmann, G.: Spin model checker, the: primer and reference manual. Addison-Wesley Professional, Reading (2003)

11. Kesten, Y., Piterman, N., Pnueli, A.: Bridging the gap between fair simulation and trace inclusion. In: Hunt Jr., W.A., Somenzi, F. (eds.) CAV 2003. LNCS, vol. 2725, pp. 381-392. Springer, Heidelberg (2003)

12. Kesten, Y., Pnueli, A., Raviv, L., Shahar, E.: LTL model checking with strong fairness. Formal Methods in System Design (2002)

13. Cadence Berkeley Lab. Cadence SMV (1998), http://www-cad.eecs.berkeley . edu/kenmcmil/smv

14. Nielson, J.L.: Buddy, http://buddy . sourceforge . net

15. Piterman, N.: Suggested projects (2009), http: / /www. doc .ic.ac.uk/ npiterma/ projects.html

16. Piterman, N., Pnueli, A.: Faster solutions of rabin and streett games. In: LICS, pp. 275-284 (2006)

17. Piterman, N., Pnueli, A., Sa'ar, Y.: Synthesis of reactive(1) designs. In: Emerson, E.A., Namjoshi, K.S. (eds.) VMCAI 2006. LNCS, vol. 3855, pp. 364-380. Springer, Heidelberg (2005)

18. Pnueli, A., Shahar, E.: A platform for combining deductive with algorithmic verification. In: Alur, R., Henzinger, T.A. (eds.) CAV 1996. LNCS, vol. 1102, pp. 184-195. Springer, Heidelberg (1996)

19. Ranjan, R.K., Sanghavi, J.V., Brayton, R.K., Vincentelli, A.S.: High performance BDD package based on exploiting memory hierarchy. In: DAC'96, June 1996, pp. 635-640 (1996)

20. Sa'ar, Y.: JTLV - web API, http://jtlv.ysaar.net/resources/javaDoc/ API1.3.21

21. Somenzi, F.: CUDD: CU Decision Diagram package (1998), http: / /vlsi . colorado . edu/ fabio/CUDD/

22. Whaley, J.: JavaBDD, http: //javabdd. sourceforge.net

23. Wongpiromsarn, T., Topcu, U., Murray, R.M.: Automatic synthesis of robust embedded control software. submitted to AAAI'10 (2010)

24. Wongpiromsarn, T., Topcu, U., Murray, R.M.: Receding horizon control for temporal logic specifications. In: HSCC'10 (2010) 